\title{
Para no salir(se) de cauce: la regulación emocional en el trabajo docente
}

\author{
Guillermo Martínez Cuevas
}

\section{Resumen}

La labor docente expone al profesorado a situaciones que pueden generar un amplio abanico de emociones, las cuales influyen en el desempeño y bienestar de maestros y estudiantes. El objetivo de este trabajo es presentar estrategias que ayuden a regular dichas emociones, a partir de la propuesta de Gross y Thompson. Se plantea la importancia de generar programas de formación de los profesores sobre el tema y de acudir a tratamiento especializado cuando las emociones permanecen a pesar de los intentos de regulación.

Palabras clave: emociones, regulación emocional, docencia, salud mental, autocuidado.

\section{NOT GOING BEYOND THE LIMITS: EMOTION REGULATION IN THE TEACHER'S WORK}

\begin{abstract}
The teaching profession exposes teachers to situations that can trigger a set of different emotions, which can influence students' and teachers' performance and well-being. The aim of this paper is to present strategies that can help to regulate those emotions, based on Gross and Thompson's proposal. It suggests the importance of developing teacher's training programmes on this topic and turn to specialized treatment when negative emotional reactions persist despite regulation attempts.
\end{abstract}

Keywords: emotions, emotion regulation, teaching, mental health, self-care. 


\section{Guillermo Martínez Cuevas}

Licenciado en Pedagogía, Maestro en Psicología Clínica, Maestro en Ciencias en el campo de la Salud Mental Pública y profesor de carrera en el en el área interdisciplinaria "Psicología y Educación" del Colegio de Pedagogía de la Facultad de Filosofía y Letras (UNAM). Sus líneas de trabajo son formación docente, educación socioemocional, resiliencia, educación para la salud y promoción de la salud mental. Es fundador del curso "Promoción de la salud mental en contextos escolares" que se imparte cada verano en el Instituto Nacional de Psiquiatría "Ramón de la Fuente Muñiz" desde 2013.

\section{Introducción}

El trabajo docente implica la relación con una gran variedad de emociones, las cuales pueden influir en el bienestar del profesorado, su rendimiento y la relación con sus estudiantes: experiencias de ilusión, frustración, ira, alegría, preocupación, miedo o tristeza pueden llegar a ser difíciles de manejar. Gross y Thompson (2007) señalan que la regulación emocional consiste en los esfuerzos que hace una persona, de manera consciente o inconsciente, para influir en la intensidad y la duración de sus emociones, así como en la manera de expresarlas.

A pesar de que las emociones se presentan constantemente en el trabajo docente, existen pocas opciones para formar a los maestros sobre este tema (Palomera, Briones y Gómez-Linares, 2017). Un estudio reciente de tipo cualitativo entre profesores de nivel bachillerato y licenciatura en la Universidad Nacional Autónoma de México (UnAm), concluyó que es necesario que los programas de formación y profesionalización de los docentes promuevan, entre otros aspectos, el bienestar del docente, tanto en su desarrollo humano como en sus derechos a la salud física, psicológica y social (de Agüero, et al., 2019).

El objetivo de este trabajo es presentar un conjunto de estrategias que ayuden a regular las emociones que pueden llegar a presentarse en el ejercicio docente; para ello servirá de sustento la propuesta de Gross y Thompson sobre regulación emocional, la cual, debido a los diversos factores que incluye, se ha vuelto una de las más populares (Gómez y Calleja, 2016). Me dirigiré en particular a los docentes de educación superior, quienes, desde mi experiencia, requieren

${ }^{1}$ El autor desea agradecer a la Lic. Cynthia Lima Cruz por sus comentarios técnicos al texto. enormemente este tipo de información, aunque lo que aquí se menciona también es útil para profesores de otros niveles educativos. ${ }^{1}$ 


\section{¿Necesitan los docentes herramientas para la regulación emocional?}

Sus emociones pueden motivarme (cuando se ven alegres, entusiasmados,
etc.) o desmotivarme (cuando se ven molestos); a veces, también he llega-
do a sentirme angustiada porque los veo decaídos o muy cansados.

Alumna, 22 años, refiriéndose a sus maestros de la licenciatura.

Regular las emociones tiene impacto en una de las metas más importantes para el docente, que es lograr el aprendizaje. Éste podría verse favorecido si en los estudiantes se disminuyen las emociones negativas, especialmente el aburrimiento y la frustración, ya que atenuando estas dos, se aumentan las emociones positivas (Ochoa de Alda, et al., 2019).

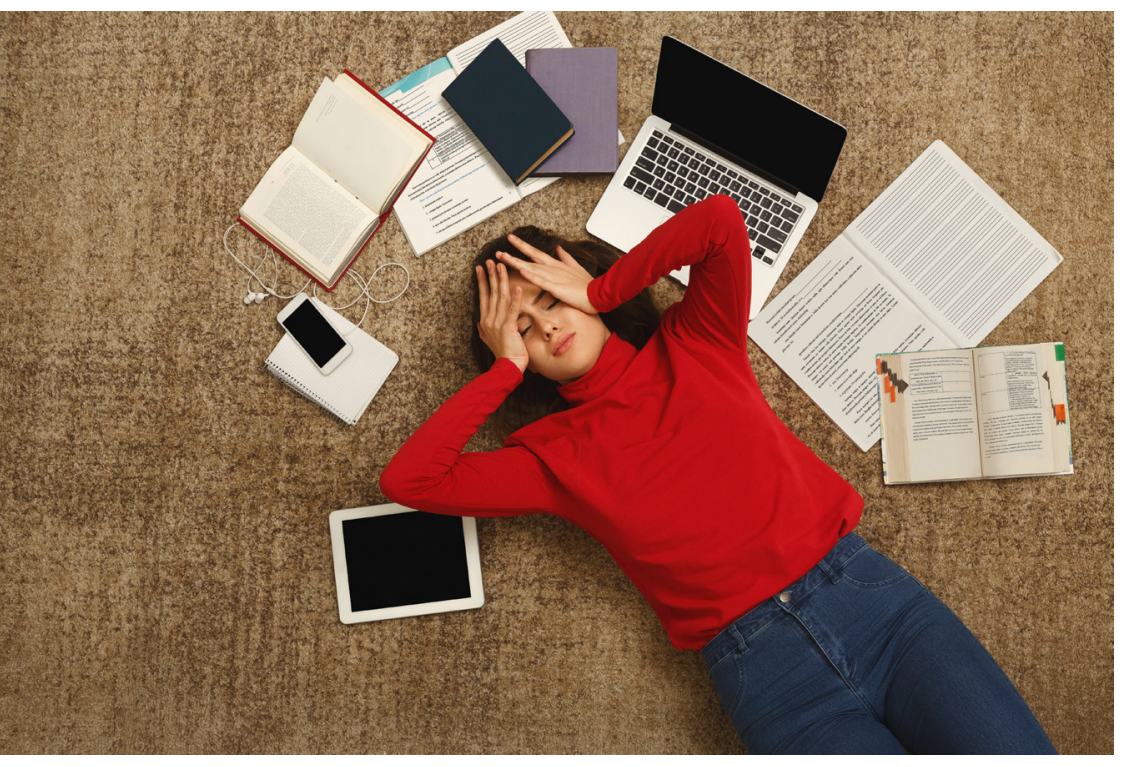

Además de ello, la regulación emocional es sumamente importante para el cuidado del propio docente. Las profesiones de servicio humano, como la docencia (junto con las del cuidado de la salud, los servicios sociales, las profesiones religiosas o de orientación), implican relacionarse permanentemente con los sentimientos de ira, vergüenza, desesperanza o frustración de los beneficiarios del servicio. En el caso de la docencia, con las emociones de los estudiantes; asimismo, en esta clase de trabajos, las normas institucionales prevalecientes llevan a la falta de atención en uno mismo al anteponer las necesidades de los otros, a trabajar durante muchas horas y a hacer todo lo que sea necesario para ayudar. Aunado a ello, otras características del trabajo, tales como la sobrecarga, la insuficiencia de recompensas, la falta de control y de imparcialidad, la relación con los iguales y el conflicto entre los valores personales y los organizacionales, pueden generar estrés crónico, lo que conlleva el riesgo de desarrollar el llamado síndrome de burnout, el cual se caracteriza por el agotamiento (sentirse rebasado y vacío de recursos emocionales y físicos), el cinismo (respuestas insensibles o apáticas ante diversos aspectos del trabajo o hacer sólo lo mínimo) y la sensación de ineficacia (sentirse incompetente, carente de logros y productividad en el desempeño laboral).

Existe una asociación entre presentar burnout y diversos síntomas físicos, tales como dolores de cabeza, alteraciones gastrointestinales, tensión muscular, episodios de resfríos o gripe, hipertensión y alteraciones del sueño (Maslach, 2009; Maslach y Jackson, 1981). Además del malestar personal, también hay 
influencia sobre los alumnos; aquellos docentes que presentan síndrome de burnout promueven la baja motivación de los estudiantes (Shen, et al., 2015). De ahí que los docentes requieren desarrollar su capacidad de regulación emocional para cuidar de sí mismos, y también para estar atentos en la promoción o eliminación de cierto tipo de emociones entre los estudiantes, favoreciendo así el aprendizaje.

\section{Regulación emocional: las estrategias de Gross y Thompson}

En este trabajo se empleará la clasificación de emociones utilizada por Gross (2014) quien utiliza la palabra "afecto" para englobar:

1) Emociones (como la ira o la tristeza)

2) Respuestas al estrés

3) Humor (como la depresión o la euforia).

Gross (2014) explica que el "estrés" se refiere típicamente a afectos negativos o inespecíficos, mientras que la "emoción" puede incluir estados afectivos positivos y negativos; por otra parte, las "emociones" pueden distinguirse del "humor", pues éste dura más que aquellas; además de que las emociones suelen ser provocadas por objetos específicos y dan lugar a conductas relevantes hacia estos objetos; en contraste, el humor es más difuso e influye más en los pensamientos que en las conductas.

De igual manera que se habla de "afectos" como una gran categoría, también se hace referencia a la "regulación de los afectos", la cual se refiere a todos los esfuerzos por influir en la carga afectiva de una respuesta, incluyendo: 1) la regulación de la emoción; 2) la regulación del estrés, y 3) la regulación del estado de ánimo. Lo que comparten estos tres tipos de regulación es que tienen como meta el maximizar el placer o minimizar el dolor. No se sabe hasta dónde la regulación de la emoción, del estrés o del estado de ánimo son más similares que diferentes; por ello, es conveniente poner atención al tipo de afecto que desea regularse. Para Lazarus (2000) existe una interdependencia entre emociones, estrés y estrategias para tratar de afrontar el estrés (en inglés coping), ya que coexisten como un todo. ${ }^{2}$

A partir de estas consideraciones, Gross y Thompson (2007) proponen las siguientes estrategias de regulación emocional:

${ }^{2}$ A propósito de esta complejidad, Gross (1998), señala que el estudio de la regulación emocional abarca las distintas disciplinas de la psicología (biológica, cognitiva, del desarrollo, social, de la personalidad, clínica y de la salud) para dar explicaciones que se complementan mutuamente.

a) Elegir una situación. Tomar acciones que hacen que sea más - o menosprobable estar en una situación de la cual se derivan emociones deseables o indeseables; por ejemplo, que un padre de familia elija una peluquería donde sea más probable que su hijo "tolere" que le corten el cabello.

b) Modificación de una situación. Esta estrategia se enfoca en generar esfuerzos para modificar la situación en sí misma, o bien, alterar el impacto emocional que tiene dicha situación. Esto puede realizarse cambiando el medio ambiente físico, pero también expresando las emociones, ofreciendo ayuda o validando las emociones de otros (en vez de denigrarlas o castigarlas). 
c) Despliegue de la atención. También es posible regular la emoción sin cambiar el medio ambiente. El despliegue de la atención se refiere a cómo los individuos dirigen su atención en una situación dada, con el fin de influir en sus emociones, por ejemplo: focalizar la atención en diferentes aspectos de la situación o bien traer a la memoria recuerdos o pensamientos diferentes a la situación emocional desagradable.

d) Cambio cognoscitivo. Esta estrategia se refiere a modificar la manera en que evaluamos o apreciamos una situación, para así alterar su importancia, modificando la forma de pensar en ella, o bien valorando la propia capacidad para manejar las demandas de esa situación, por ejemplo, comparando la circunstancia actual con una más compleja ya vivida.

e) Modulación de la respuesta. Esta estrategia se enfoca en influir en respuestas fisiológicas, conductas o experiencias, tan directamente como sea posible; algunas personas buscan sentir alivio tratando de relajar los músculos, comiendo, haciendo ejercicio, bebiendo alcohol o fumando tabaco; todo ello busca modificar la experiencia emocional.

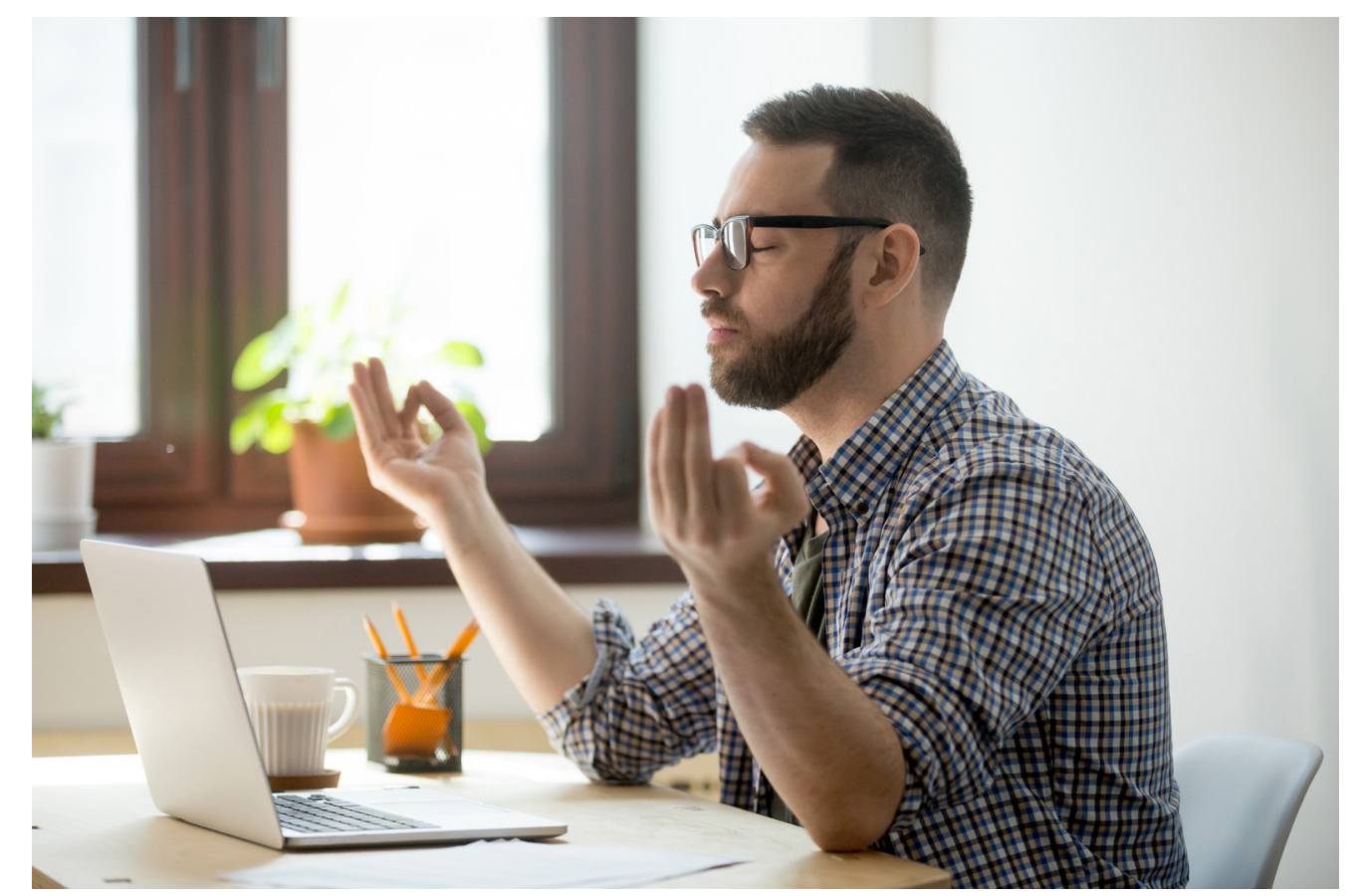

Algunasideas para aplicar lo propuesto por GrossyThompson

Si eres paciente en un momento de ira, escaparás a cien días de tristeza.

Proverbio chino

El esquema de Gross y Thompson (2007) aquí presentado puede ayudarnos a identificar nuevas estrategias y a valorar la importancia de las ya conocidas. A continuación, se enunciarán diversas sugerencias en el marco de esta propuesta teórica. 
a) Elegir la situación (tratar de controlar las probabilidades de que una emoción se produzca). Resulta de enorme utilidad considerar la planeación cuidadosa de cada una de las clases. Para evitar el aburrimiento es muy útil incorporar la variedad (por ejemplo, cambiando el entorno físico o alternando la incorporación de imágenes con sonidos); la curiosidad (preguntando al grupo: “qué pasaría si...?”); y la sorpresa (incorporando un evento inesperado). Para evitar la sensación de frustración, será importante presentar los contenidos de forma clara y organizada, promover la repetición y el repaso, dividir las tareas complejas en unidades más simples, además de presentar a los estudiantes modelos (a través de la proyección de un video, o la modelación que puede hacer el propio profesor u otro estudiante) que ejecuten aquellas actividades que deben aprender los estudiantes, observando los procedimientos paso a paso; además de reconocer y reforzar el avance académico de los estudiantes cada vez que éstos se presenten (Woolfolk, 2006). Los programas de formación para mejorar la eficacia de los docentes también impactan en las emociones de los profesores, pues éstos pueden sentirse más confiados y entusiastas (Steinert, et al., 2016).

b) Modificar la situación (modificar el espacio, expresar emociones u ofrecer ayuda). Es conveniente que el docente esté atento a mejorar, en la medida de lo posible, las condiciones de iluminación, ventilación, etcétera. Sin embargo, también es muy útil que el docente aprenda a expresar sus emociones empleando los "mensajes yo", que significa hablar en primera persona, por ejemplo "yo opino...", "a mí me parece que...", "yo me siento..." (Comas, et al., 2002; Acero, et al., 2004). Los "mensajes yo" son lo opuesto a los "mensajes tú", que comienzan con la frase "tú siempre... te distraes, me haces enojar". Se podría utilizar una fórmula como la siguiente: 1) "yo me siento___ 2) cuando tú___ 3) por lo que te pido___. Así, en vez de decir "te distraes con el teléfono", puede decirse: "yo me siento molesto cuando sacas el teléfono porque pierdo el hilo de lo que estoy diciendo, por lo que te pido que lo saques solamente cuando sea necesario para la clase". Igualmente, este modelo sirve para reconocer las conductas positivas: "yo me siento muy contento cuando ustedes participan en clase, por lo que les pido que continúen leyendo el material y sigan expresando sus opiniones". En este sentido se ha recomendado el entrenamiento de los profesores en la habilidad de recompensar las conductas adecuadas de los estudiantes, para fomentar la conducta positiva, el rendimiento escolar y la formación de lazos en la escuela (Instituto Nacional sobre el Abuso de Drogas, 2004). Otro aspecto muy importante es lograr la disciplina grupal sin autoritarismo dentro del aula: un maestro que carece de habilidades para relacionarse con los alumnos disruptivos puede desarrollar síntomas de burnout, mientras que uno que logra tener un buen control disciplinario desarrolla un sentido personal de logro y competencia laboral (Evers, et al., 2004).

c) Desplegar la atención (aprender a manejar la atención). Durante los últimos años el desarrollo de programas de mindfulness, o atención plena, han 
${ }^{3}$ Una manera sencilla de iniciar el desarrollo de la atención puede encontrarse en la siguiente liga: https:// www.youtube.com/ watch? $v=Y$ JBB8ambUdl\& $\mathrm{t}=25 \mathrm{~s}$.

${ }^{4}$ Puede conocer un poco más acerca de ella revisando este video:

https://www.youtube.com/ watch? $\mathrm{v}=-\mathrm{FgClO} \mathrm{iY} \mathrm{Q} \mathrm{Qc}$.

${ }^{5}$ Un ejemplo de aplicación está en el siguiente video: https://www.youtube.com/ watch?v=vjgyy $1 \mathrm{bpvO} 4$ mostrado una gran utilidad; dichos programas consisten en el aprendizaje de prácticas de meditación (desligadas de aspectos religiosos). Una de sus características principales consiste en tratar de dirigir la atención al aquí y al ahora amablemente, sin que los participantes se juzguen a sí mismos si durante su práctica aparecen pensamientos distractores. Existe evidencia de que esta técnica ha ayudado a profesores de distintos niveles educativos a disminuir los niveles de estrés y los días de baja laboral (Mañas, Franco y Justo, 2011), así como a disminuir los síntomas de burnouty a desarrollar la capacidad de atención (Flook, et al., 2013).3

d) Cambio cognoscitivo (reevaluación). Una idea o la interpretación de lo que sucede en el salón de clase es capaz de despertar en nosotros una emoción profunda. El filósofo griego Epicteto (1802) señalaba: "No son las cosas las que turban a los hombres, sino la opinión que de ellas forman". Es posible que juzguemos la realidad a partir de ciertos patrones de pensamiento más o menos automáticos, sin detenernos a pensar si son ciertos. Es importante detectar las emociones negativas y los pensamientos que generan esas emociones para reestructurarlos, tratando de ser más objetivos y realistas, y debido a ello, sentirse mejor (Bimbela y Navarro, 2007). Un docente puede sentir malestar cuando piensa: "no voy a poder explicarlo bien y se van a desesperar"; "están viendo el celular para jugar". Si se ponen en duda esos pensamientos, pueden reestructurarse por otras interpretaciones tales como: "lo que quiero transmitir es importante y lo he hecho antes. He aprendido cada vez más. Voy a comentarles que si algo no se entiende, pueden preguntar y me detendré a explicarlo nuevamente"; "algunos se distraerán con el celular, pero me ha sucedido que otros estudiantes están verificando en internet cosas que he dicho para profundizar en el tema".

e) Modulación de la respuesta (influir en las respuestas fisiológicas, experiencias o conductas tan directamente como sea posible). Al sentirnos alterados, la respiración se hace superficial, lo que provoca mareos, dolor de cabeza y sensación de tensión, entre otros. Para disminuir esta activación es eficaz tratar de respirar profundamente y de la forma más pausada posible; para lograrlo es importante utilizar el diafragma, un músculo que se encuentra debajo de los pulmones, de manera que, al tensarlo y relajarlo, se aprovecha toda la capacidad respiratoria. Al usar el diafragma, la zona del abdomen se infla al inspirar, y se desinfla al expirar. Es normal que al tratar de practicarlo se tenga cierta dificultad, ya que usualmente se respira empleando la musculatura de los hombros y el pecho (Bimbela y Navarro, 2007). A esta técnica se le conoce como respiración diafragmática. ${ }^{4}$

Para los estudiantes también resultan muy difíciles diversos aspectos de la vida académica, entre ellos la sobrecarga de trabajo, la participación en clase (Pozos-Radillo, et al., 2015) y, por supuesto, las evaluaciones (JerezMendoza y Oyarzo-Barría, 2015). Dedicar algunos minutos en el aula para enseñarles a atender a las respuestas fisiológicas que sufren en esas situaciones, les ayudará a regular sus emociones. ${ }^{5}$ 
${ }^{6}$ Puede consultar la página de la Organización Mundial de la Salud: https://bit.ly/2qZRNDn Igualmente, sobre el tema del burnout en docentes, el siguiente video es muy ilustrativo:

https://www.youtube.com/ watch?v=D67m6clMeuY

${ }^{7}$ La distimia se define como un trastorno afectivo crónico que persiste por lo menos dos años en adultos y un año en adolescentes y niños (liménezMaldonado, et al., 2013: 212).

${ }^{8}$ Sobre el tema, puede encontrarse más información en la página de la Organización

Mundial de la Salud: https:// www.who.int/campaigns/worldhealth-day/2017/es/.

Y también se sugieren los siguientes videos: https:// www.youtube.com/

watch?v=FIEDhEmcLP4\&t $=14 \mathrm{~s}$

y https://www.youtube.com/ watch? $v=H Z$ W6m2jAHuw.

\section{Cuando las emociones permanecen}

En ocasiones el estudio personal y/o la capacitación en estrategias de regulación no son suficientes para hacer sentir mejor a los docentes y se requiere la intervención de profesionales. Sentirse exhausto, impotente, desesperanzado, desinteresado, insatisfecho, pueden ser indicadores de burnout. Según diversos estudios, dicho síndrome se puede presentar entre el 7\% y el 52\% de los profesores universitarios (Martínez, 2016). Recientemente la Organización Mundial de la Salud ha incluido a este síndrome en la Clasificación Internacional de Enfermedades, con el nombre de "Síndrome de desgaste ocupacional". 6

Hay que considerar que la presencia constante de emociones como la tristeza, el enojo, la apatía, la desesperanza, la sensación de vacío, la culpa, el sentirse "distraído", la preocupación, la inquietud, el miedo e incluso el pánico, junto con otros síntomas, pueden clasificarse como trastornos de ansiedad o trastornos del afecto; entre estos últimos, se encuentran la depresión y la distimia7 (Asociación Americana de Psiquiatría, 2013). Diversos estudios realizados en docentes universitarios señalan que la presencia de ansiedad en este sector va desde un 7\% hasta un 37\%; en otros estudios, la depresión en docentes universitarios se ha detectado desde un 8\% y hasta un 52\% (Martínez y Keller, 2019). Tanto la depresión como la ansiedad son resultado de interacciones biológicas, psicológicas y sociales, por lo que no es suficiente "echarle ganas" para salir adelante. El médico especialista dedicado a atender la ansiedad y la depresión es el psiquiatra, aunque también algunos médicos generales se han capacitado para atender estas afecciones. Otros especialistas son los psicólogos clínicos, que por medio de la psicoterapia abordan patrones de pensamiento y conducta que generan malestar en el profesorado. También algunos pedagogos y trabajadores sociales proporcionan psicoeducación, es decir, explican a las personas, de manera individual o grupal, las características de su malestar, así como estrategias de autocuidado. ${ }^{8}$

\section{Conclusiones}

He experimentado una gran diversidad de emociones que van del encanto al fastidio. De la esperanza al desaliento. De la confianza a la desilusión. Todo ello se debe a la propia empatía, la participación y sentimientos de los alumnos, pero también a las condiciones laborales y políticas institucionales.

Profesor de licenciatura. Once años de experiencia.

El trabajo docente implica la relación constante con las emociones propias y las de los estudiantes. La regulación emocional consiste en los esfuerzos por acortar ciertas emociones y promover o extender otras, así como en la posibilidad de expresarlas; su importancia radica en que mediante ella se promueve el bienestar y el aprendizaje. La propuesta de Gross y Thompson (2007) proporciona un marco para comprender las distintas acciones que pueden tomarse para regular las emociones: tratar de anticiparse a situaciones que 


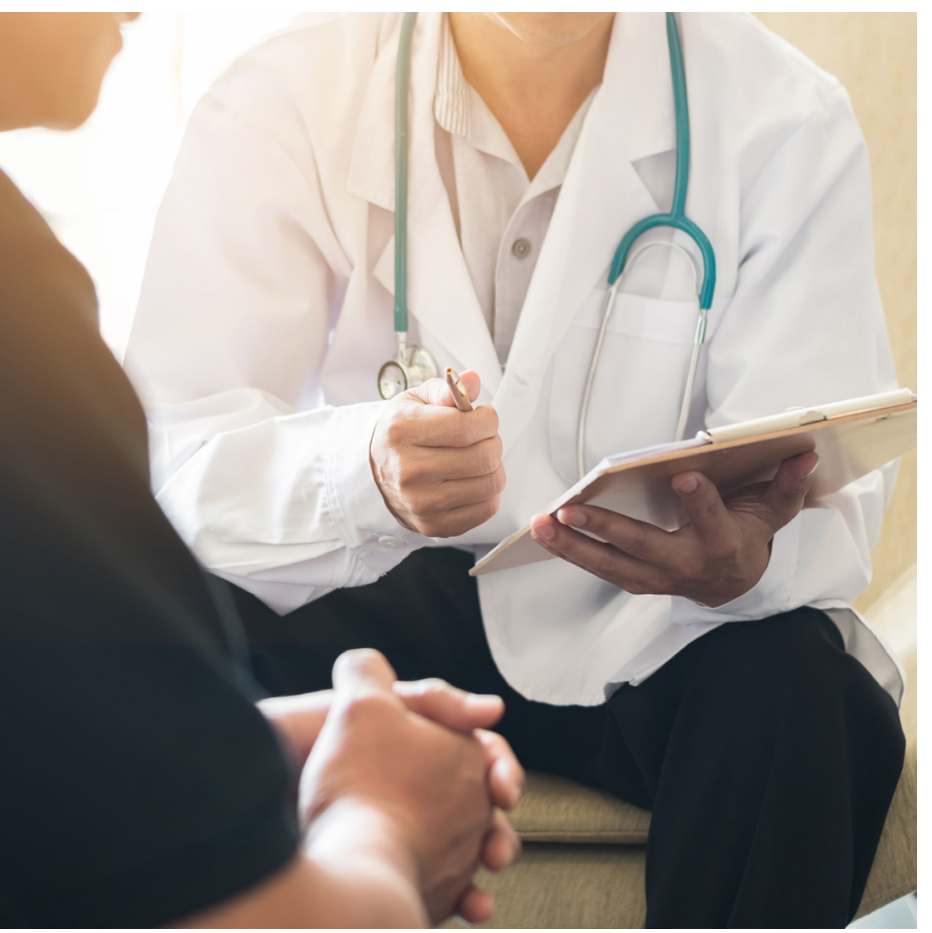

\section{Referencias}

* Acero, A., Moreno, G. y Moreno, J. (2004). Programa de aprendizaje y desarrollo de habilidades sociales "Atenea". Cuaderno del alumno. Madrid: Deporte y Vida, Asociación de Deportistas.

* Asociación Americana de Psiquiatría. (2013). Guía de consulta de los criterios diagnósticos del DSM 5. Arlington, VA: Asociación Americana de Psiquiatría.

* Bimbela, J. L. y Navarro, B. (2007). Cuidando al formador. Habilidades emocionales y de comunicación (2a ed.). Granada, España: Escuela Andaluza de Salud Pública. Consejería de Salud.

* Comas, R., Moreno, G. y Moreno, J. (2002). Programa Ulises: Aprendizaje y desarrollo del autocontrol emocional. Madrid: Asociación Deporte y Vida.

* de Agüero, M., Torres, R., Olvera, A., Lara, Y. A., Pompa, M. y Martínez, A. M. d. P. (2019). La formación y profesionalización de las profesoras y los profesores de la UNAM para la enseñanza universitaria. Un estudio cualitativo con grupos focales para la enseñanza universitaria. In M. Sánchez y A. M. d. P. Martínez (Eds.), Formación docente en la UNAM: antecedentes y la voz de su profesorado (pp. 241-307). México: Universidad Nacional Autónoma de México. Coordinación de Desarrollo e Innovación Curricular.

Epicteto. (1802). Manual de Epicteto. In. Madrid.

- Evers, W. J., Tomic, W. y Brouwers, A. (2004). Burnout among teachers: Students' and teachers' perceptions compared. School Psychology International, 25(2), 131-148. 
* Flook, L., Goldberg, S. B., Pinger, L., Bonus, K. y Davidson, R. J. (2013). Mindfulness for teachers: A pilot study to assess effects on stress, burnout, and teaching efficacy. Mind, Brain, and Education, 7(3), 182-195.

* Gross, J. J. (1998). The emerging field of emotion regulation: An integrative review. Review of general psychology, 2(3), 271-299.

* Gross, J. J. (2014). Emotion Regulation: Conceptual and Empirical Foundations. In J. J. Gross (Ed.), Handbook of Emotion Regulation (Second ed., pp. 3-20). United States of America: The Guildford Press.

* Gross, J. J. y Thompson, R. A. (2007). Emotion Regulation. Conceptual Fundations. In J. J. Gross (Ed.), Handbook of Emotion Regulation (pp. 3-24). United States of America: The Guildford Press.

* Gómez, O. y Calleja, N. (2016). Regulación emocional: definición, red nomológica y medición. Revista mexicana de investigación en psicología, 8(1), 96-117.

* Instituto Nacional sobre el Abuso de Drogas. (2004). Cómo prevenir el uso de drogas en los niños y los adolescentes. Una guía con base científica para padres, educadores y líderes de la comunidad. (Segunda ed.).

* Jiménez-Maldonado, M. E., Gallardo-Moreno, G. B., Villaseñor-Cabrera, T. y González-Garrido, Andrés A. (2013). La distimia en el contexto clínico. Revista Colombiana de Psiquiatría. 42(2), 212-218.

* Jerez-Mendoza, M. y Oyarzo-Barría, C. (2015). Estrés académico en estudiantes del Departamento de Salud de la Universidad de Los Lagos Osorno. Revista chilena de neuro-psiquiatría, 53(3), 149-157.

* Lazarus, R. S. (2000). Estrés y emoción. Manejo e implicaciones en nuestra salud. Bilbao, España: Editorial Desclée de Brouwer, S.A.

* Martínez, G. (2016). Identificación de malestar emocional y síndrome de burnout en docentes universitarios. (Maestría en Ciencias de la Salud). Universidad Nacional Autónoma de México, Ciudad de México. Retrieved from http://132.248.9.195/ ptd2016/noviembre/0753432/Index.html

* Martínez, G. y Keller, J. (2019). Promoción de la salud mental: una necesidad ante los síntomas de ansiedad, depresión y síndrome de burnout en el profesorado universitario. In A. Valero (Ed.), Promoción, alfabetización e intervención en salud: experiencias desde la multidisciplina (1ra. ed., pp. 115-129).

* Maslach, C. (2009). Comprendiendo el burnout. Ciencia \& Trabajo, 11(32), 37-43.

* Maslach, C. y Jackson, S. E. (1981). The measurement of experienced burnout. Journal of organizational behavior, 2(2), 99-113.

* Mañas, I., Franco, C. y Justo, E. (2011). Reducción de los Niveles de Estrés Docente y los Días de Baja Laboral por Enfermedad en Profesores de Educación Secundaria Obligatoria a través de un Programa de Entrenamiento en Mindfulness. Clínica y salud, 22(2), 121-137. 
- Ochoa de Alda, J. A. G., Marcos-Merino, J. M., Méndez, F. J., Mellado, V. y Esteban, M. R. (2019). Emociones académicas y aprendizaje de biología, una asociación duradera. Enseñanza de las ciencias: revista de investigación y experiencias didácticas, 37(2), 43-61.

* Palomera, R., Briones, E.y Gómez-Linares, A. (2017). Diseño, desarrolloy resultados de un programa de educación socio-emocional para la formación de docentes a nivel de grado y postgrado. Contextos educativos: Revista de educación(20), 165-182.

* Pozos-Radillo, B. E., Preciado-Serrano, M. d. L., Plascencia, A. R., Acosta-Fernández, M. y Aguilera, M. d. I. Á. (2015). Estrés académico y síntomas físicos, psicológicos y comportamentales en estudiantes mexicanos de una universidad pública. Ansiedad y estrés, 21(1).

- Shen, B., McCaughtry, N., Martin, J., Garn, A., Kulik, N. y Fahlman, M. (2015). The Relationship between Teacher Burnout and Student Motivation. British Journal of Educational Psychology, 85(4), 519-532.

Steinert, Y., Mann, K., Anderson, B., Barnett, B. M., Centeno, A., Naismith, L., . . . Viggiano, T. (2016). A systematic review of faculty development initiatives designed to enhance teaching effectiveness: A 10-year update: BEME Guide No. 40. Medical teacher, 38(8), 769-786.

* Woolfolk, A. (2006). Psicología Educativa (Novena ed.). México: Pearson Educación de México, S.A de C.V.

\section{Cómo citar este artículo}

- Martínez Cuevas, Guillermo (2019). Para no salir(se) de cauce: la regulación emocional en el trabajo docente. Revista Digital Universitaria (RDU). Vol. 20, núm. 6 noviembrediciembre. Dol: http://doi.org/10.22201/codeic.16076079e.2019.v20n6.a1.

Recepción: 08/08/2019. Aprobación: 19/09/2019 